\title{
GENERATION OF ANALYTIC SEMIGROUPS BY STRONGLY ELLIPTIC OPERATORS
}

\author{
BY
}

\section{H. BRUCE STEWART}

ABSTRACT. Strongly elliptic operators realized under Dirichlet boundary conditions in unbounded domains are shown to generate analytic semigroups in the topology of uniform convergence. This fact is applied to initial-boundary value problems for temporally homogeneous and temporally inhomogeneous parabolic equations.

Introduction. The theory of continuous semigroups of operators, when combined with information about strongly elliptic operators, provides useful results about parabolic equations. For example, let $A$ be a strongly elliptic operator of order $2 m$ on a domain $\Omega$, with zero Dirichlet boundary conditions. If an estimate

$$
\left\|(-A-z)^{-1}\right\|<M /|z|
$$

is established for complex $z$ in a sector $|\arg z|<1 / 2 \pi+\epsilon$, then the parabolic equation

$$
(\partial / \partial t) u+A u=0
$$

has solutions which are analytic in $t$.

Such resolvent estimates for $A$ have been established in the Banach spaces $L^{p}$. In [5], Browder proved an estimate like (E) in $L^{2}$; Agmon, in [1], gave an argument which proves $(\mathrm{E})$ in $L^{p}$, although [1] deals explicitly with the distribution of eigenvalues and the completeness of eigenfunctions. A theorem in $L^{p}$ is stated in Friedman [7].

In the space $C$ of continuous functions with the topology of uniform convergence, not so much has been known. Eidelmann, in [6], discusses the fundamental solution of parabolic equations more general than (P); Arima extends the work, in [4], to obtain a solution to a general initial-boundary value problem. However, the estimates in [6] and [4] are not strong enough near the boundary to prove the analyticity of semigroups (unless there is no boundary, i.e., $\Omega=\mathbf{R}^{N}$ ).

Received by the editors November 26, 1973.

AMS (MOS) subject classifications (1970). Primary 35K35, 47D05. 
Recently Masuda, in [14a], presented a method of obtaining the resolvent estimate (E) for second-order elliptic operators with real coefficients and zero Dirichlet boundary conditions, in the space $C$. The present paper extends Masuda's results to higher order strongly elliptic operators.

Masuda has since announced similar results for higher order operators, in [14b]; in this work, as in [14a], Masuda studies in detail fundamental solutions of the operator $A+z$. The present paper uses a somewhat easier method which promises straightforward generalizations (to more general boundary conditions, and estimates in $C^{\mu}$ ). These will be the subject of a second paper.

$\S 1$ of this paper sets forth the notation and basic assumptions, and states the main elliptic estimate to be proved. $\$ 2$ establishes this estimate for operators with constant coefficients, on the whole space or half-space; $\S 3$ proceeds to the general case. $\$ 4$ verifies all the conditions for generation of analytic semigroups. $\S 5$ offers simple applications to temporally homogeneous and temporally inhomogeneous parabolic differential equations, showing the analyticity in $t$ of the solutions.

The author wishes to express his indebtedness to Professor Tosio Kato for his generous guidance and important suggestions; also to Professors C. B. Morrey, Jr., and $\mathrm{H}$. O. Cordes for helpful discussions.

1. Main estimates. To prove that an elliptic operator $A$ generates an analytic semigroup, one needs estimates for the resolvent of $-A$. This section gives the precise statement of the estimates obtained.

We make several assumptions about the operator $A$, similar to some of those used by Browder in [5].

Let the differential operator $A$ be defined on an open set $\Omega$ in $\mathbf{R}^{N}$ :

$$
A=\sum_{|\alpha| \leqslant 2 m} a_{\alpha}(x) D^{\alpha}, \quad D^{\alpha}=\frac{\partial^{\alpha_{1}}}{\partial x_{1}^{\alpha_{1}}} \frac{\partial^{\alpha_{2}}}{\partial x_{2}^{\alpha_{2}}} \cdots \frac{\partial^{\alpha_{N}}}{\partial x_{N}^{\alpha_{N}}}, \quad x \in \Omega \subset \mathbf{R}^{N} .
$$

Separating the highest order terms of $A$, we define

$$
p(x ; \xi)=(-1)^{m} \sum_{|\alpha|=2 m} a_{\alpha}(x) \xi^{\alpha}, \quad x \in \Omega, \xi \in \mathbf{R}^{N} .
$$

About the operator $\boldsymbol{A}$ we will assume that:

$A$ satisfies the roots condition: for $x \in \partial \Omega, \xi^{\prime}, \eta \in \mathbf{R}^{N}$ tangent and normal, respectively, to $\partial \Omega$ at $x$, the polynomial in $\lambda, p\left(x ; \xi^{\prime}+\lambda \eta\right)$ has exactly $m$ roots with positive imaginary part.

$$
\text { The } a_{\alpha} \text { are measurable and uniformly bounded on } \bar{\Omega} \text { by } L \text {. }
$$


(A4),

For $|\alpha|=2 m$, the $a_{\alpha}$ are uniformly continuous

on $\Omega$, with modulus of continuity $\omega(|x|)$.

REMARK. It is well known that (A2) follows from (A1) if $N>2$, or if the coefficients of $p(x ; \xi)$ are real.

The domain $\Omega$ will be an open set with boundary which is uniformly regular of class $C^{2 m}$; this means that

There is a collection $\left\{N_{k}, \Phi_{k}\right\}$ of open sets and mappings of the open sets onto $B(0,1)=\{y:|y|<1\}$. $\Phi_{k}$ maps

$$
\begin{gathered}
N_{k} \cap \Omega \text { onto }\left\{y:|y|<1, y_{N}>0\right\} \\
N_{k} \cap \partial \Omega \text { onto }\left\{y:|y|<1, y_{N}=0\right\}
\end{gathered}
$$

(B2) The sets $\Phi_{k}^{-1}(B(0,1 / 2))$ cover an $\epsilon$-neighborhood of the boundary $\partial \Omega$.

There is a number $K$ such that the intersection of any $K+1$ distinct sets $N_{k}$ is empty.

The mappings $\Phi_{k}$ are one-to-one, and they and their inverse functions

(B4) have derivatives up to order $2 m$ which are continuous and bounded uniformly in $k$ by $L^{\prime}$.

The set $\Omega$ will be locally regular of class $C^{2 m, \mu}$ if, in addition, the derivatives of $\Phi_{k}$ and its inverse satisfy a uniform Hölder condition of order $\mu>0$ on $N_{k}$. This stronger assumption is first used in $\S 4$.

Various norms and seminorms will be used in our estimates:

$$
\begin{aligned}
\|u\|_{L^{p}(\Omega)} & =\left(\int_{\Omega}|u(x)|^{p} d x\right)^{1 / p}, & |[u]|_{j, L^{p}(\Omega)} & =\sum_{|\alpha|=j}\left\|D^{\alpha} u\right\|_{L^{p}(\Omega)}, \\
\|u\|_{W^{j, p}(\Omega)} & =\sum_{0 \leqslant i \leqslant j}|[u]|_{i, L^{p}(\Omega)}, & \|u\|_{C(\Omega)} & =\sup _{x \in \Omega}|u(x)|, \\
|[u]|_{j, C(\Omega)} & =\sum_{|\alpha|=j}\left\|D^{\alpha} u\right\|_{C(\Omega)}, & \|u\|_{C^{j}(\Omega)} & =\sum_{0 \leqslant i<j} \mid\left[u \|_{i, C(\Omega)} .\right.
\end{aligned}
$$

The normed space $W^{j, p}(\Omega)$ is the completion in its norm of the space $C^{\infty}(\bar{\Omega})$ of infinitely differentiable functions (if $\left.j \leqslant 2 m\right) . C^{j}(\Omega)$ is the space of functions with bounded, continuous derivatives on $\Omega$ up to order j. $W_{\text {loc }}^{j, p}(\Omega)$ and $C_{\text {loc }}^{j}(\Omega)$ consist of functions which are in $W^{i, p}(\Omega \cap B)$ and $C^{j}(\Omega \cap B)$ respectively, for every closed bounded set $B \subset \Omega$. The space $C^{j, \mu}(\Omega)$ consists of functions in $C^{j}(\Omega)$ whose $j$ th order derivatives satisfy a uniform Hölder condition of order $\mu$ on $\Omega$. 
The differential operators will act in the special sets of functions:

$C_{0}(\bar{\Omega})=\{u$ continuous and bounded on $\bar{\Omega}$, with $u(x) \rightarrow 0$ as $|x| \rightarrow \infty\}$, $D_{0}^{q}(A)=\left\{u \in C_{0}(\bar{\Omega})\right.$, with $A u \in C_{0}(\bar{\Omega}), u \in W_{\text {loc }}^{2 m, q}(\bar{\Omega})$,

$$
\text { and } \left.D^{\beta} u=0 \text { on } \partial \Omega, 0 \leqslant|\beta|<m\right\} \text {. }
$$

The main estimate to be proved is:

THEOREM 1. Let $q>N$. Then under the above assumptions on $A$ and $\Omega$, there are positive constants $\epsilon, M_{0}, \lambda_{0}, r_{0}$ depending on $q, E, L, \omega, \Omega$, such that for $u \in D_{0}^{q}(A)$

$$
\|u\|_{C(\Omega)}+\sum_{0<|\beta|<2 m}|z|^{-|\beta| / 2 m}\left\|D^{\beta} u\right\|_{C(\Omega)}
$$

$$
\begin{aligned}
&+\sum_{|\gamma|=2 m}|z|^{-1+\theta} \sup _{x_{0} \in \Omega}\left\|D^{\gamma} u\right\|_{L} q_{\left(B\left(x_{0}, r_{z}\right) \cap \Omega\right)} \\
& \leqslant\left(M_{0} /|z|\right)\|(A+z) u\|_{C(\Omega)}
\end{aligned}
$$

for $|z| \geqslant \lambda_{0}, \operatorname{larg} z \mid \leqslant 1 / 2 \pi+\epsilon$. Here $\theta=N / 2 m q, r_{z}=r_{0}|z|^{-1 / 2 m}$.

Theorem 1 follows directly from:

THEOREM 2. Under the circumstances of Theorem 1

$$
\begin{aligned}
& \sum_{0<|\beta|<2 m}|z|^{|\beta| / 2 m}\left\|D^{\beta} u\right\|_{C(\Omega)} \\
&+\sum_{|\gamma|=2 m}|z|^{-1+\theta} \sup _{x_{0} \in \Omega}\left\|D^{\gamma} u\right\|_{L q_{\left(B\left(x_{0}, r_{z}\right) \cap \Omega\right)}} \\
& \leqslant M_{0}|z|^{-1+\theta} \sup _{x_{0} \in \Omega}\|(A+z) u\|_{L} q_{\left(B\left(x_{0}, r_{z}\right) \cap \Omega\right)}
\end{aligned}
$$

Proof of Theorem 1 using Theorem 2. To establish (1.1), examine the right-hand side of (1.2), and notice that, due to the choice of $r_{z}$,

$$
\begin{aligned}
& |z|^{-1+\theta}\|(A+z) u\|_{L^{q}\left(B\left(x_{0}, r_{z}\right) \cap \Omega\right)} \\
& \leqslant|z|^{-1+\theta}\|(A+z) u\|_{C(\Omega)}\left(\operatorname{meas} B\left(x_{0}, r_{z}\right)\right)^{1 / q} \\
& \leqslant \text { const }|z|^{-1+\theta}|z|^{(N / 2 m) \cdot(1 / q)}\|(A+z) u\|_{C(\Omega)} \\
& \leqslant \text { const }|z|^{-1}\|(A+z) u\|_{C(\Omega)} \text {. Q.E.D. }
\end{aligned}
$$

2. Constant coefficients. By a process of localization, Theorem 2 will be reduced to the following simple case:

(a) The operator $A$ is replaced by an operator $A^{\#}$ having constant coefficients and only derivatives of order $2 m ; A^{\#}$ satisfies conditions (A1), (A2), and $(\mathrm{A} 3)$ of $\S 1$. 
(b) The domain is either $\mathbf{R}^{N}$ or $\mathbf{R}_{+}^{N}=\left\{x: x_{N}>0\right\}$.

First we give a pair of preliminary lemmas. The first of the pair states a priori estimates proved by Agmon, Douglis, and Nirenberg in [3].

Lemma 1. Let $1<p<\infty$. Suppose $T$ is a differential operator with constant coefficients $a_{\alpha}$ only of order $2 m$, which satisfies the roots condition (A2) and the inequality

$$
\left|\sum_{|\alpha|=2 m} a_{\alpha} \xi^{\alpha}\right| \geqslant E^{\prime}|\xi|^{2 m}
$$

and $\left|a_{\alpha}\right|<L$.

Then there is a constant $C$ depending on $p, E^{\prime}, L$, such that:

(i) for all $u \in W^{2 m, p}\left(\mathbf{R}^{N}\right)$ with compact support

$$
\|u\|_{W^{2 m, p}\left(\mathrm{R}^{N}\right)} \leqslant C\left\{\|T u\|_{L^{p}\left(\mathrm{R}^{N}\right)}+\|u\|_{L^{p}\left(\mathrm{R}^{N}\right)}\right\}
$$

(ii) for all $u \in W^{2 m, p}\left(\mathbf{R}_{+}^{N}\right)$ with compact support, and satisfying $D^{\beta} u=0$, $|\beta|<m$, on $x_{N}=0$,

$$
\|u\|_{W^{2 m, p_{\left(\mathrm{R}_{+}^{N}\right)}^{N}}} \leqslant C\left\{\|T u\|_{L^{p_{\left(\mathrm{R}_{+}^{N}\right)}^{N}}}+\|u\|_{L^{p_{\left(\mathrm{R}_{+}^{N}\right.}^{N}}}\right\} .
$$

Proof. These estimates are essentially proved in Theorems $14.1^{\prime}$ and 14.1 of Agmon, Douglis, Nirenberg [3].

The next lemma will yield the $|z|$ dependence of the a priori estimates for $A^{\#}+z$.

Lemma 2. Let $1<p<\infty$. Suppose $A^{\#}$ is a constant coefficient differential operator with terms only of order $2 m$, and $z$ is a complex number. Let $G=\mathbf{R}^{N}$ or $\mathbf{R}_{+}^{N}$.

Suppose $u \in W^{2 m, p} .(G)$. Let $y=\rho^{-1 / 2 m} x$, and define $u^{\prime}(y)=\rho^{-1} u(x)$, where $\rho>0$. Then

and

$$
\left\|D^{\beta} u\right\|_{L^{p}(G)}=\rho^{1-|\beta| / 2 m+N / 2 m p}\left\|D^{\beta} u^{\prime}\right\|_{L^{p}(G)}, \quad|\beta| \leqslant 2 m,
$$

$$
\left\|\left(A^{\#}+z\right) u\right\|_{L^{p}(G)}=\rho^{N / 2 m p}\left\|\left(A^{\#}+\rho z\right) u^{\prime}\right\|_{L^{p}(G)} .
$$

If $u \in C^{2 m-1}(\bar{G})$,

$$
\left\|D^{\beta} u\right\|_{C(\bar{G})}=\rho^{1-|\beta| / 2 m}\left\|D^{\beta} u^{\prime}\right\|_{C(\bar{G})}, \quad|\beta| \leqslant 2 m-1 .
$$

Here the differentiations and integrations are always in the coordinates of the function mentioned, i.e., $x$ for $u, y$ for $u^{\prime}$.

Proof. We notice that a scale change of variables takes $\mathrm{R}^{N}$ onto $\mathrm{R}^{N}$ and $\mathbf{R}_{+}^{N}$ onto $\mathbf{R}_{+}^{N}$. 
(2.6) follows easily from the definitions; (2.4) requires a change of variable of integration. For (2.5), observe that

$$
\begin{aligned}
\left(A^{\#+z) u}\right. & =\left(\sum_{|\alpha|=2 m} a_{\alpha} D_{x}^{\alpha}+z\right) u(x) \\
& =\left(\sum_{|\alpha|=2 m} a_{\alpha} D_{y}^{\alpha} \rho^{-|\alpha| / 2 m}+z\right) \rho u^{\prime}(y)=\left(A^{\#}+\rho z\right) u^{\prime} .
\end{aligned}
$$

Then again the change of variable of integration leads to (2.5). Q.E.D.

Lemma 3 gives the $L^{p}$ estimates for $\left(A^{\#}+z\right)$.

Lemma 3. Let $1<p<\infty$. Suppose (a) and (b) above hold. Then there are positive constants $\epsilon, C$, depending on $p, E, L$, such that for $|z|>0$, $|\arg z| \leqslant 1 / 2 \pi+\epsilon$ :

(i) for all $u \in W^{2 m, p}\left(\mathbf{R}^{N}\right)$

$$
\sum_{0<j<2 m}|z|^{1-j / 2 m}|[u]|_{j, L}{\left(\mathrm{R}^{N}\right)} \leqslant C\left\|\left(A^{\#}+z\right) u\right\|_{L^{p}\left(\mathrm{R}^{N}\right)}
$$

(ii) for all $u \in W^{2 m, p}\left(\mathbf{R}_{+}^{N}\right)$ with $D^{\beta} u=0,|\beta|<m$, on $x_{N}=0$,

$$
\sum_{0 \leqslant j \leqslant 2 m}|z|^{1-j / 2 m}|[\mu]|_{j, L}^{p}\left(\mathrm{R}_{+}^{N}\right) \leqslant C\left\|\left(A^{\#}+z\right) u\right\|_{L^{p}\left(\mathrm{R}_{+}^{N}\right)} \cdot
$$

PROof. The method is essentially that of Agmon [1] but uses the a priori estimates for $\mathbf{R}^{N}$ or $\mathbf{R}_{+}^{N}$ instead of for a bounded domain.

Consider the differential operator in $N+1$ variables

$$
T=A^{\#}+(-1)^{m} e^{i \theta}(\partial / \partial t)^{2 m}
$$

with which we associate the polynomial $p(\xi)+e^{i \theta} \tau^{2 m}$. The operator $T$ is elliptic, and one can show that

$$
\left|p(\xi)+e^{i \theta} \tau^{2 m}\right| \geqslant E^{\prime}
$$

for $|\xi|^{2}+|\tau|^{2}=1$ and $|\theta| \leqslant 1 / 2 \pi+\epsilon$, with $E^{\prime}, \epsilon>0$ depending on $E, L$. This follows from the assumptions (A1) and (A3), which imply that there is a $C>0$ with $|\operatorname{Im} p(\xi)| \leqslant C|\xi|^{2 m} \leqslant C / E \operatorname{Re}(p(\xi))$ and hence there is $\epsilon>0$ depending on $E, L$ such that $|\arg p(\xi)| \leqslant 1 / 2 \pi-2 \epsilon$.

Let $G$ be $\mathbf{R}^{N}$. or $\mathbf{R}_{+}^{N}$, and define

$\Gamma=\{(x, t): x \in G, t \in \mathbf{R}\}, \Gamma_{0}=\{(x, t): x \in G, t \in(-1 / 2,1 / 2)\}$.

To apply the a priori estimates of Lemma 1 , it remains only to verify the root condition for $T$ at the boundary of $\Gamma$ : for $\xi^{\prime}$ tangent, $\eta$ normal to $\partial \Omega$, the polynomial $p\left(\xi^{\prime}+\lambda \eta\right)+e^{i \theta}\left(\tau^{\prime}\right)^{2 m}$ has exactly $m$ roots $\lambda$ with positive imaginary part.

But connecting the points $p\left(\xi^{\prime}+\lambda \eta\right)+e^{i \theta}\left(\tau^{\prime}\right)^{2 m}$ and $p\left(\xi^{\prime}+\lambda \eta\right)$ by a line in the complex plane, a standard continuity argument reduces the question to 
the root condition for $p\left(\xi^{\prime}+\lambda \eta\right)$, which is satisfied by hypothesis.

Now we can apply Lemma 1 to obtain an a priori estimate for the operator $T$. Consider the class of functions $v \in C^{2 m}(\bar{\Gamma})$ with compact support and satisfying Dirichlet boundary conditions zero ( $x$ derivatives only) if $G=R_{+}^{N}$. For such functions Lemma 1 implies

$$
\|v\|_{2 m, L}^{p}(\Gamma) \leqslant C\left(\|T v\|_{L^{p}(\Gamma)}+\|v\|_{L^{p}(\Gamma)}\right)
$$

where $C$ depends on $p, E^{\prime}, L$.

In particular, let

$$
v=v_{\lambda}(x, t)=\phi(t) e^{i \lambda t} u(x), \quad \lambda \text { real, }
$$

where $\phi \in C_{0}^{\infty}(\mathrm{R})$ is 1 for $|t| \leqslant 1 / 2$ and zero for $|t| \geqslant 1$; and $u(x) \in C^{2 m}(\bar{G})$ has compact support and satisfies null Dirichlet boundary conditions if $G=\mathbf{R}_{+}^{N}$. Then

$$
\left\|v_{\lambda}\right\|_{2 m, L^{p}(\Gamma)} \geqslant\left\|e^{i \lambda t} u\right\|_{2 m, L}^{p}\left(\Gamma_{0}\right) \cdot
$$

Also,

$$
T v_{\lambda}=T\left(\phi e^{i \lambda t} u\right)=\phi T\left(e^{i \lambda t} u\right)+\cdots=\phi e^{i \lambda t}\left(A^{\#}+\lambda^{2 m} e^{i \theta}\right) u+\cdots
$$

where the remaining terms involve only the function $u$ itself, and derivatives of $\phi$ which are bounded. In fact, we find that

$$
\begin{aligned}
& \left(\left\|T v_{\lambda}\right\|_{L^{p}(\Gamma)}+\left\|v_{\lambda}\right\|_{L^{p}(\Gamma)}\right) \\
& \quad \leqslant C\left\{\left\|\left(A^{\#}+\lambda^{2 m} e^{i \theta}\right) u\right\|_{L^{p}(G)}+\lambda^{2 m-1}\|u\|_{L^{p}(G)}\right\} .
\end{aligned}
$$

Also

$$
\begin{aligned}
\left\|e^{i \lambda t} u\right\|_{2 m, L}^{p}\left(\Gamma_{0}\right) & \geqslant \int_{G} \sum_{k+|\alpha|<2 m}|\lambda|^{p k}\left|D^{\alpha} u\right|^{p} d x \\
& \geqslant \int_{G} \sum_{|\alpha|=j}|\lambda|^{p(2 m-j)}\left|D^{\alpha} u\right|^{p} d x, \quad j \leqslant 2 m \\
& =|\lambda|^{p(2 m-j)}|[u]|_{j, L^{p}(G)^{\circ}}^{p}
\end{aligned}
$$

Combining these results with the a priori estimate, we have

$$
\begin{aligned}
& \sum_{0<j<2 m}|\lambda|^{2 m-j}|[u]|_{j, L}{ }^{p}(G) \\
& \leqslant(2 m+1) C\left\|\left(A^{\#}+\lambda^{2 m} e^{i \theta}\right) u\right\|_{L^{p}(G)}+(2 m+1) C \lambda^{2 m-1}\|u\|_{L^{p}(G)} .
\end{aligned}
$$

Choosing $\lambda$ large enough that $1 / 2 \lambda \geqslant(2 m+1) C$, we can substract the last term from both sides of the inequality, and get, with a new $C$,

$$
\sum_{0 \leqslant j \leqslant 2 m}|\lambda|^{2 m-j}|[u]|_{j, L^{p}(G)} \leqslant C\left\|\left(A^{\#}+\lambda^{2 m} e^{i \theta}\right) u\right\|_{L^{p}(G)} .
$$


Setting $z=\lambda^{2 m} e^{i \theta},(2.7)$ and (2.8) follow for compactly supported $u \in C^{2 m}(G)$ for large $|z|$. For $u \in W^{2 m, p}(G)$, the result follows by completion in $W^{2 m, p}(G)$.

For small $|z|$, one may use Lemma 2, and (2.4) and (2.5) in particular. The dilation factor $\rho$ transforms the problem to the case of large $|z|$. Q.E.D.

Now we use Lemma 3 to establish corresponding estimates involving $C$ norms.

Lemma 4. Let $q>N$. Suppose (a) and (b) of this section hold. Define $\theta=N / 2 m q$.

Then there are positive constants $\epsilon, C$ depending on $q, E, L$, such that for $|z|>0, \operatorname{larg} z \mid<1 / 2 \pi+\epsilon$ :

(i) for all $u \in W^{2 m, q}\left(\mathbf{R}^{N}\right)$,

$$
\begin{aligned}
& \sum_{0<j \leqslant 2 m-1}|z|^{1-j / 2 m}|[u]|_{j, C\left(\mathrm{R}^{N}\right)}+|z|^{\theta}|[u]|_{2 m, L}{ }^{q}\left(\mathrm{R}^{N}\right) \\
& \leqslant C|z|^{\theta}\left\|\left(A^{\#}+z\right) u\right\|_{L^{q}\left(\mathrm{R}^{N}\right)}
\end{aligned}
$$

(ii) for all $u \in W^{2 m, q}\left(\mathbf{R}_{+}^{N}\right)$ with $D^{\beta} u=0,|\beta|<m$, on $x_{N}=0$,

$$
\begin{aligned}
\sum_{0<j \leqslant 2 m-1}|z|^{1-j / 2 m}|[u]|_{j, C\left(\mathrm{R}_{+}^{N}\right)}+|z|^{\theta}|[u]|_{2 m, L}{ }_{\left(\mathrm{R}_{+}^{N}\right)} & \leqslant C|z|^{\theta}\left\|\left(A^{\#}+z\right) u\right\|_{L^{q}\left(\mathrm{R}_{+}^{N}\right)} . \\
& \leqslant
\end{aligned}
$$

Proof. Fixing $z=z_{0},\left|z_{0}\right|=1$, and $q=p$ in Lemma 3, we find that for $\left|\arg z_{0}\right| \leqslant 1 / 2 \pi+\epsilon$,

$$
\sum_{0<j \leqslant 2 m}\left|\left[u^{\prime}\right]\right|_{j, L^{q}} \leqslant C\left\|\left(A^{\#}+z_{0}\right) u^{\prime}\right\|_{L^{q}} .
$$

But by the Sobolev theorem, since $q>N$,

$$
\sum_{0 \leqslant j \leqslant 2 m-1}\left|\left[u^{\prime}\right]\right|_{j, C} \leqslant C \sum_{0 \leqslant j \leqslant 2 m}\left|\left[u^{\prime}\right]\right|_{j, L} q
$$

and so for $\left|z_{0}\right|=1,\left|\arg z_{0}\right| \leqslant 1 / 2 \pi+\epsilon$, we can say

$$
\sum_{0<j \leqslant 2 m-1}\left|\left[u^{\prime}\right]\right|_{j, C}+\left|\left[u^{\prime}\right]\right|_{2 m, L} q \leqslant C\left\|\left(A^{\#}+z_{0}\right) u^{\prime}\right\|_{L^{q}} .
$$

The dependence on $|z|$ can now be obtained using Lemma 2. Let $z$ be a complex number, $\rho>0$, and $\rho z=z_{0}$. Let $u$ be as in the hypotheses of the present lemma, $u^{\prime}$ defined as in Lemma 2. Substitutions from Lemma 2 yield the result. Q.E.D.

3. Proof of main estimate. The estimates of Lemma 4 are the essential ingredients in proving Theorem 2 . The proof proceeds by reduction to local estimates. One establishes the proper estimates for $A+z$ locally; then the supremum is taken. 
Proof of Theorem 2. First we will establish local estimates for an operator $\bar{A}$, on a ball or a half-ball, with variable coefficients $\bar{a}_{\alpha}(y)$. This operator is not the same as $A$, but has the same properties (A1)-(A4); $\bar{A}$ will shortly be obtained from $A$ by local change of coordinates.

Let $G=B\left(y_{0}, \delta\right)$ or $G=B\left(y_{0}, \delta\right) \cap \mathbf{R}_{+}^{N}$, with $y_{0}$ on the edge of $\mathbf{R}_{+}^{N}$. Suppose $v \in W^{2 m, q}\left(\mathbf{R}^{N}\right)$ or $v \in W^{2 m, q}\left(\mathbf{R}_{+}^{N}\right)$, has support on $\bar{G}$, and satisfies null Dirichlet boundary conditions in the case of the half-ball. Choosing

$$
\bar{A}^{\#}=\sum_{|\alpha|=2 m} \bar{a}_{\alpha}\left(y_{0}\right) D^{\alpha},
$$

Lemma 4 implies

$$
\begin{aligned}
\sum_{0 \leqslant j \leqslant 2 m-1}|z|^{1-j / 2 m}|[v]|_{j, C(G)}+|z|^{\theta}|[v]|_{2 m, L^{q}(G)} & \\
& \leqslant C|z|^{\theta}\left\|\left(\bar{A}^{\#}+z\right) v\right\|_{L^{q}(G)} .
\end{aligned}
$$

Note that $C$ depends on $q, E, L$, but not on $y_{0}$ or $\delta$.

If $\bar{A}$ satisfies (A1)-(A4), we write

$$
\begin{aligned}
\left(\bar{A}^{\#}+z\right)= & \sum_{|\alpha|=2 m} \bar{a}_{\alpha}\left(y_{0}\right) D^{\alpha}+z \\
= & \sum_{|\alpha| \leqslant 2 m} \bar{a}_{\alpha}(y) D^{\alpha}+z-\sum_{|\alpha|<2 m} \bar{a}_{\alpha}(y) D^{\alpha} \\
& -\sum_{|\alpha|=2 m}\left(\bar{a}_{\alpha}(y)-\bar{a}_{\alpha}\left(y_{0}\right)\right) D^{\alpha}
\end{aligned}
$$

and hence

$$
\begin{aligned}
\left\|\left(\bar{A}^{\#}+z\right) v\right\|_{L^{q}(G)} \leqslant & \|(\bar{A}+z) v\|_{L^{q}(G)}+C \sum_{0 \leqslant j<2 m-1}|[v]|_{j, L^{q}(G)} \\
& +\sup _{y \in G,|\alpha|=2 m}\left|\bar{a}_{\alpha}(y)-\bar{a}_{\alpha}\left(y_{0}\right) \|[v]\right|_{2 m, L} q_{(G)} .
\end{aligned}
$$

Since

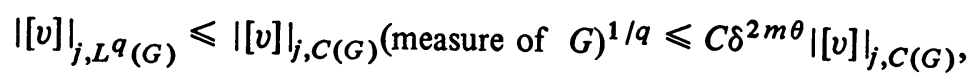

(3.2) now becomes

$$
\begin{aligned}
& \sum_{0<j \leqslant 2 m-1}|z|^{1-j / 2 m}|[v]|_{j, C(G)}+|z|^{\theta}|[v]|_{2 m, L^{q}(G)} \\
& \leqslant C|z|^{\theta}\left\{\|(\bar{A}+z) v\|_{L^{q}(G)}+\delta^{2 m \theta} \sum_{0<j<2 m-1}|[v]|_{j, C(G)}\right. \\
& \left.\quad+\sup _{y \in G,|\alpha|=2 m}\left|\bar{a}_{\alpha}(y)-\bar{a}_{\alpha}\left(y_{0}\right) \|[v]\right|_{2 m, L} q(G)\right\}
\end{aligned}
$$


Now we specify restrictions on the values of $\delta$ and $|z|$. Choose:

$$
\begin{gathered}
0 .<\delta \leqslant \delta_{0} \text { s.t. }\left|y-y_{0}\right|<\delta_{0} \Rightarrow\left|\bar{a}_{\alpha}(y)-\bar{a}_{\alpha}\left(y_{0}\right)\right| \leqslant 1 / 2 C, \\
|\alpha|=2 m,|z| \geqslant \lambda_{0}>1 \text { s.t. } \lambda_{0}^{1 / 2 m} \geqslant 2 C \lambda_{0}^{\theta} \delta_{0}^{2 m \theta} .
\end{gathered}
$$

The first condition allows the third term in braces on the right of (3.3) to be subtracted from both sides. Following the second condition,

$$
|z|^{1-j / 2 m-\theta} \geqslant|z|^{1 / 2 m-\theta} \geqslant \lambda_{0}^{1 / 2 m-\theta} \geqslant 2 C \delta_{0}^{2 m \theta}
$$

or

$$
|z|^{1-j / 2 m} \geqslant 2 C|z|^{\theta} \delta^{2 m \theta}
$$

and hence the second group of terms in braces on the right of (3.3) can also be subtracted from both sides. By adjusting $C$, we then have

$$
\begin{aligned}
\sum_{0<j<2 m-1}|z|^{1-j / 2 m}|[v]|_{j, C(G)}+|z|^{\theta}|[v]|_{2 m, L} q_{(G)} \\
\leqslant C|z|^{\theta}\|(\bar{A}+z) v\|_{L^{q}(G)} .
\end{aligned}
$$

Now we wish to use the mappings described in assumptions (B1)-(B4) to obtain estimates near the curved boundary of $\Omega$. Suppose that $x_{0} \in \Phi_{k}^{-1}(B(0,1 / 2))$. Due to assumption (B4), we may choose an $r_{0}$, depending on $L^{\prime}$ but not on $k$ or $x_{0}$, so small that $B\left(x_{0}, r_{0}\right) \subset N_{k}$ and:

$$
\begin{aligned}
& \text { If } 0 \leqslant s \leqslant 1, r=s r_{0}, \delta=s \delta_{0}, \\
& \Phi_{k}\left(B\left(x_{0}, r\right) \cap \Omega\right) \subset B\left(y_{0}, \delta\right) \cap \mathbf{R}_{+}^{N} \text { for some } y_{0} .
\end{aligned}
$$

Here $y_{0}$ may be chosen on the edge of $\mathbf{R}_{+}^{N}$ so long as $B\left(x_{0}, r\right)$ meets $\partial \Omega$.

Let $u \in W^{2 m, q}(\Omega)$ have support in $B\left(x_{0}, r\right)$; define $y=\Phi_{k}(x)$ and $v(y)=u(x)$. The image of $A$ is a new operator $\bar{A}, A(u(x))=\bar{A}(v(y))$. It is well known that $\bar{A}$ is an elliptic operator satisfying (A1)-(A4) with $E, L, \omega$ adjusted by multiples of $L^{\prime}$. Therefore (3.5) is mapped over to

$$
\begin{aligned}
\sum_{0<j \leqslant 2 m-1}|z|^{1-j / 2 m}|[u]|_{j, C\left(G^{\prime}\right)}+|z|^{\theta}|[u]|_{2 m, L^{q}\left(G^{\prime}\right)} \\
\leqslant C|z|^{\theta}\|(A+z) u\|_{L^{q}\left(G^{\prime}\right)}
\end{aligned}
$$

where $C$ depends on $q, E, L, \omega$, and $L^{\prime}$.

The estimate on $\Omega$ can now be developed from the local estimate (3.7). Suppose $u \in D_{0}^{q}(A)$, and choose a localizing function $\phi \in C_{0}^{\infty}\left(\mathbf{R}_{+}^{N}\right)$ which is nonnegative, identically 1 on $B(0,1 / 2)$, and identically zero outside $B(0,1)$. For any $x_{0} \in \bar{\Omega}$, define $\phi_{0}(x)=\phi\left(\left(x-x_{0}\right) / r\right)$. Then $\phi_{0} u \in W^{2 m, q}\left(\mathbf{R}^{N}\right)$ or $\phi_{0} u \in W^{2 m, q}(\Omega)$, has support in $B\left(x_{0}, r\right)$, and satisfies null Dirichlet boundary conditions on $\partial \Omega$. Let $G^{\prime}=B\left(x_{0}, r\right) \cap \Omega$, and $G^{\prime \prime}=B\left(x_{0}, 1 / 2 r\right) \cap \Omega$. Then 
from (3.7) we have

$$
\begin{aligned}
\sum_{0 \leqslant j \leqslant 2 m-1}|z|^{1-j / 2 m}|[u]|_{j, C\left(G^{\prime \prime}\right)}+|z|^{\theta}|[u]|_{2 m, L^{q}\left(G^{\prime \prime}\right)} \\
\quad \leqslant \sum_{0 \leqslant j \leqslant 2 m-1}|z|^{1-j / 2 m}\left|\left[\phi_{0} u\right]\right|_{j, C\left(G^{\prime}\right)}+|z|^{\theta}\left|\left[\phi_{0} u\right]\right|_{2 m, L^{q}\left(G^{\prime}\right)} \\
\quad \leqslant C|z|^{\theta}\left\|(A+z) \phi_{0} u\right\|_{L^{q}\left(G^{\prime}\right)^{\circ}}
\end{aligned}
$$

But

$$
(A+z) \phi_{0} u=\phi_{0}(A+z) u+\sum_{|\alpha| \leqslant 2 m, \beta<\alpha} a_{\alpha}\left(\begin{array}{l}
\alpha \\
\beta
\end{array}\right) D^{\alpha-\beta} \phi_{0} D^{\beta} u .
$$

Since, for $r<1,\left|D^{\alpha-\beta} \phi_{0}\right| \leqslant C r^{|\beta|-|\alpha|}$, we arrive at

$$
\begin{aligned}
& \sum_{0<j \leqslant 2 m-1}|z|^{1-j / 2 m}|[u]|_{j, C\left(G^{\prime \prime}\right)}+|z|^{\theta}|[u]|_{2 m, L^{q}\left(G^{\prime \prime}\right)} \\
& \quad \leqslant C|z|^{\theta}\left\{\|(A+z) u\|_{L^{q}\left(G^{\prime}\right)}+\sum_{0 \leqslant j \leqslant 2 m-1} r^{-2 m+j+2 m \theta}|[u]|_{j, C\left(G^{\prime}\right)}\right\} .
\end{aligned}
$$

To obtain (1.2), we now take the sup of (3.8) over all $x_{0} \in \bar{\Omega}$. This gives

$$
\sum_{0 \leqslant j \leqslant 2 m-1}|z|^{1-j / 2 m}|[u]|_{j, C(\Omega)}+|z|^{\theta} \sup _{x_{0} \in \Omega}|[u]|_{2 m, L}{ }_{\left(B\left(x_{0}, 1 / 2 r\right) \cap \Omega\right)}
$$

$$
\begin{aligned}
\leqslant C|z|^{\theta}\left\{\sup _{x_{0} \in \Omega}\|(A+z) u\|_{L^{q}\left(B\left(x_{0}, r\right) \cap \Omega\right)}\right. & \\
& \left.+\sum_{0<j \leqslant 2 m-1} r^{-2 m+j+2 m \theta}|[u]|_{j, C(\Omega)}\right\}
\end{aligned}
$$

The final choice of $r$ can now be made. Pick $r_{0}, \delta_{0}$, and $\lambda_{0}$ in accordance with (3.6) and (3.4); and furthermore let $\lambda_{0}$ be large enough that

$$
\lambda_{0}^{1 / 2 m} \geqslant 2 C \lambda_{0}^{\theta} r_{0}^{-2 m+j+2 m \theta} \text {. }
$$

For any particular $z$ with. $|z| \geqslant \lambda_{0}$, set

$$
r=2 r_{z}=r_{0}|z|^{-1 / 2 m} \text {. }
$$

Such a choice of $r$ is appropriate to (3.6) and (3.4). Furthermore, we can now subtract the second set of terms in braces on the right of (3.9) from both sides.

In order to put (3.9) in the precise form of (1.2), we note that any set $B\left(x_{0}, r\right) \cap \Omega$ can be covered by a fixed finite number of sets of the form $B\left(x_{0}, 1 / 2 r\right) \cap \Omega$, and hence 


$$
\sup _{x_{0} \in \bar{\Omega}}\|(A+z) u\|_{L^{q}\left(B\left(x_{0}, r\right) \cap \Omega\right)} \leqslant C \sup _{x_{0} \in \bar{\Omega}}\|(A+z) u\|_{L^{q}\left(B\left(x_{0}, 1 / 2 r\right) \cap \Omega\right)} \text { Q.E.D. }
$$

REMARK. Similarly one can prove an $L^{p}$ estimate for $A+z$ under the same assumptions on $A$ and $\Omega$ (including $\Omega$ unbounded). In fact, one could prove Lemma 3 directly in this general case.

However, for Theorem 2, even in the simplest case $A=A^{\#}, \Omega=\mathbf{R}^{N}$, a localization argument is necessary. This is because in order to derive Theorem 1 from Theorem 2, the right-hand side of (1.2) must involve $L^{q}$ norms over small bounded sets.

4. Generation of semigroups. Define the following realization of $A: A_{0}^{q}$ is the operator with domain $D_{0}^{q}(A) \subset C_{0}(\bar{\Omega})$ and range in $C_{0}(\bar{\Omega})$, given by $A_{0}^{q} u=A u$ for $u \in D_{0}^{q}(A)$.

In order to prove that $A_{0}^{q}$ generates an analytic semigroup, we will need to slightly strengthen the assumptions on $A$ and $\Omega$. We introduce the condition:

The $a_{\alpha}$ can be extended outside $\Omega$ so that (A1)-(A4) are satisfied on an $\epsilon$-neighborhood of $\bar{\Omega}$.

REMARK. (A5) is automatically true if $\partial \Omega$ is compact.

THEOREM 3. Let $A$ satisfy conditions (A1)-(A4), with $\Omega$ a domain of class $C^{2 m}$. Then $A_{0}^{q}$ is a closed operator for $q>N$.

Proof. Let $\left\{u_{j}\right\} \subset D_{0}^{q}(A)$, with $\left\{u_{j}\right\}$ and $\left\{A u_{j}\right\}$ being Cauchy in the sup norm. Say $u_{j} \rightarrow v \in C_{0}(\Omega)$. By Theorem 1 , applied to $(A+z)\left(u_{j}-u_{k}\right)$ for some specific $z$, the sequences $D^{\beta} u_{j},|\beta| \leqslant 2 m-1$, are Cauchy in sup norm, hence $v$ has bounded continuous derivatives up to order $2 m-1$. Also the sequences $D^{\gamma} u_{j},|\gamma|=2 m$, are Cauchy in $L^{q}\left(B\left(x_{0}, r_{z}\right) \cap \Omega\right)$ for $x_{0} \in \bar{\Omega}$, so $v \in W_{\text {loc }}^{2 m} g(\bar{\Omega})$ and $A v=\lim A u_{j}$ a.e. where the right side is a function which belongs to $C_{0}(\bar{\Omega})$. Hence we may take $A v \in C_{0}(\bar{\Omega})$. Then $v \in D_{0}^{q}(A)$, and $A_{0}^{q}$ is a closed operator. Q.E.D.

THEOREM 4. Let (A1)-(A5) be satisfied, and let $\Omega$ be uniformly regular of class $C^{2 m}$, and locally regular of class $C^{2 m, \mu}, \mu>0$.

Suppose $q>N$. Then $A_{0}^{q}$ is a closed operator independent of $q$, which we call $A_{0}$. $\exists \lambda_{0}, \epsilon>0$ such that $A_{0}+z$ has bounded inverse on $C_{0}(\bar{\Omega})$ for $|z| \geqslant \lambda_{0}$, $\operatorname{larg} z \mid \leqslant 1 / 2 \pi+\epsilon$.

Proof. We need to show that $A_{0}^{q}+z$ maps onto $C_{0}(\bar{\Omega})$.

Due to the new assumption (A5), we can define formal operators $A^{j}$ with $C^{\infty}$ coefficients by mollifying those of $A$ : for $\phi \in C_{0}^{\infty}\left(\mathbf{R}^{N}\right)$ with $\phi \geqslant 0$ and $\int \phi d x=1$, let 


$$
\phi_{j}(x)=j^{N} \phi(j x), \quad a_{\alpha}^{j}=a_{\alpha} * \phi_{j}
$$

where $*$ indicates convolution over $\mathbf{R}^{N}$.

By standard arguments, for $j$ large enough, the resulting operators $A^{j}$ are elliptic, satisfy (A1)-(A5) with the same $E, L$, and $\omega$. For $|\alpha|=2 m, a_{\alpha}^{j} \rightarrow a_{\alpha}$ uniformly on $\Omega$; for $|\alpha|<2 m, a_{\alpha}^{j} \rightarrow a_{\alpha}$ a.e. boundedly, after possibly passing to a subsequence. We may define closed operators $\left(A^{j}\right)_{0}^{q}$.

We wish to show that the operators $\left(A^{j}\right)_{0}^{q}+\lambda$ have dense range for large real $\lambda$. For this we turn to the $L^{2}$ variational theory of elliptic operators.

For example, Agmon shows, in [2], that one can construct a bilinear form $B[u, v]$ such that for sufficiently regular functions $u, v, B[u, v]=\langle A u, v\rangle$ where $\langle$,$\rangle denotes L^{2}$ inner product over $\Omega$. Then even for unbounded domains, Gårding's inequality and the Lax-Milgram theorem can be used to show the existence of weak solutions:

There is $\lambda_{j}>0$ such that for $\lambda \geqslant \lambda_{j}, f \in C_{0}^{\infty}(\bar{\Omega})$, there is $u_{j} \in$ $H_{0}^{m}(\Omega)\left(=\right.$ completion of $C_{0}^{\infty}(\Omega)$ in the $W^{m, 2}$ norm) with

$$
A^{j} u_{j}+\lambda u_{j}=f \text { in } \Omega
$$

(Agmon [2, Theorem 8.5, Theorem 7.6, also p. 98]).

It is well known that, since the $a_{\alpha}^{j}$ and $f$ are infinitely differentiable, $u_{j}$ is also in the interior of $\Omega$. At the boundary of $\Omega$, we call upon regularity results in Morrey [15]:

$$
\begin{aligned}
& \text { Since } a_{\alpha}^{j}, f \in C^{\infty} \text {, and } \Omega \text { is locally of class } \\
& C^{2 m, \mu} \text {, the fact that } u_{j} \in H_{0}^{m}(\Omega) \text { implies that } \\
& u_{j} \in C^{2 m, \mu}(\bar{\Omega} \cap B) \text { for any bounded set } B .
\end{aligned}
$$

(Morrey [15, Theorem 6.4.8, Theorem 6.5.3]. We note that Morrey's proof is for bounded domains; but being local in nature, it applies even to bounded portions of unbounded domains.)

So we have a solution $u_{j}$ of (4.1) and the null Dirichlet boundary conditions, with $u_{j} \in H_{0}^{m}(\Omega),\left(A^{j}+\lambda\right) u_{j} \in C_{0}^{\infty}(\bar{\Omega})$, and $u_{j} \in C_{\text {loc }}^{2 m}(\bar{\Omega})$. Higher global regularity for $u_{j}$ follows from a result of Browder:

Since $u_{j}$ can be extended to a function in $C^{2 m}(N)$ for a neighborhood $N$ of any point

in $\bar{\Omega}$, if $u \in L^{p}(\Omega), f \in L^{p}(\Omega)$, then $u \in W^{2 m, p}(\Omega)$.

(Browder [5, Theorem 3].)

As an immediate consequence, $u_{j} \in W^{2 m, 2}(\Omega)$. But we can use this to show that $u_{j} \in L^{p_{0}}(\Omega)$ for some $p_{0}>2$. Then applying the above result once more gives $u_{j} \in W^{2 m, p_{0}}(\Omega)$, which in turn means $u_{j} \in L^{p_{1}}(\Omega)$ for $p_{1}>p_{0}$. 
We claim this process reaches all $p \in[2, \infty)$ in a finite number of steps. In fact, it is known (e.g. Lemma 5 of Browder [5]) that $W^{2 m, p}(\Omega) \subset L^{p^{\prime}}(\Omega)$, where $1 / p-2 m / N \leqslant 1 / p^{\prime}$. Therefore, each iteration of the process described above extends the range of $p^{-1}$ lower by a fixed amount. In a finite number of steps, the permissible values of $p^{-1}$ will include $(0,1 / 2]$, hence $p$ may be in $[2, \infty)$ as claimed.

Finally, we show $u_{j} \in D_{0}^{q}\left(A^{j}\right)$. But $C^{1}(\bar{\Omega}) \supset W^{2, p}(\Omega)$ for $p>N$, so $u_{j}$ has bounded first derivatives on $\bar{\Omega}$. Since $u_{j} \in L^{2}(\Omega)$, we must have $u_{j}(x) \rightarrow 0$ as $|x| \rightarrow \infty, u_{j} \in C_{0}(\bar{\Omega})$. Since $f \in C_{0}^{\infty}(\bar{\Omega}) \subset C_{0}(\bar{\Omega})$, (4.1) shows that $A^{j} u_{j} \in C_{0}(\bar{\Omega})$ also. Hence $u_{j} \in D_{0}^{q}\left(A^{j}\right)$.

We have just shown that for $\lambda \geqslant \lambda_{j}$, the operator $\left(A^{j}\right)_{0}^{q}+\lambda$ maps onto $C_{0}^{\infty}(\bar{\Omega})$ which is dense in $C_{0}(\bar{\Omega})$. Since $\left(A^{j}\right)_{0}^{q}+\lambda$ is a closed operator and a homomorphism, it maps onto $C_{0}(\bar{\Omega})$ by the closed range theorem.

We now interrupt the proof of Theorem 4 for the following lemma.

Lemma 5. Let $A$ be any operator for which the conclusions of Theorem 1 are valid. Let $\lambda_{0}, \epsilon$ be as in Theorem 1 , and

$$
S=\left\{z:|z| \geqslant \lambda_{0}, \arg z \mid \leqslant 1 / 2 \pi+\epsilon\right\} .
$$

Recall that $\lambda_{0}$ and $S$ depend on $q, E, L, \omega$, and $\Omega$.

Then if $A_{0}^{q}+z$ maps onto $C_{0}(\bar{\Omega})$ for some $z \in S$, the same is true for any $z \in S$.

Proof. Let $A_{0}^{q}+z_{0}$ map onto $C_{0}(\bar{\Omega})$. This means, according to the conclusions of Theorem 1, that $A_{0}^{q}+z_{0}$ has a bounded inverse. Near $z_{0}$, we can expand

$$
\left(A_{0}^{q}+z\right)^{-1}=\sum_{n=0}^{\infty}\left(A_{0}^{q}+z_{0}\right)^{-n-1}\left(z_{0}-z\right)^{n} .
$$

Now from the conclusions of Theorem $1,\left\|\left(A_{0}^{q}+z_{0}\right)^{-1}\right\|_{C} \leqslant M_{0} /\left|z_{0}\right|$, and therefore the series for $\left(A_{0}^{q}+z\right)^{-1}$ converges in an open circle about $z_{0}$ of radius $\left|z_{0}\right| / M_{0}$, or on a closed circle of radius $\left|z_{0}\right| /\left(2 M_{0}\right)$. Hence $\left(A_{0}^{q}+z\right)$ is invertible for $z$ on this closed circle.

Now let $z_{1}$ be any point on the boundary of the closed circle, provided $z_{1}$ is still in $S$. This generates a new circle, and an increased set on which $A_{0}^{q}+z$ is invertible. In this fashion, any point in $S$ may be reached by a finite chain of circles with centers $\left\{z_{i}\right\}$; for any such circle may have radius bounded from below by $\left|z_{i}\right| /\left(2 M_{0}\right) \geqslant \lambda_{0} /\left(2 M_{0}\right)$. Q.E.D.

Resuming the proof of Theorem 4, we see that $\left(A^{j}\right)_{0}^{q}+z$ is invertible for a sector depending on $q, E, L, \omega, \Omega$, associated with the operator $A$ (and hence independent of $j$ ). 
To complete the proof, we wish to show that the solutions $u_{j}$ of $A^{i} u_{j}+$ $z u_{j}=f, f \in C_{0}^{\infty}(\bar{\Omega})$, converge to a solution $u$ of

$$
A u+z u=f \text {. }
$$

The convergence of the $u_{j}$ will have to follow from the convergence of the coefficients $a_{\alpha}^{j}$.

We will use the following identity:

$$
(A+z)\left(u_{j}-u_{k}\right)=\left(A-A^{j}\right) u_{j}+\left(A^{k}-A\right) u_{k} .
$$

Now, for example,

$$
\left(A-A^{j}\right) u_{j}=\sum_{|\alpha|=2 m}\left(a_{\alpha}-a_{\alpha}^{j}\right) D^{\alpha} u_{j}+\sum_{|\alpha|<2 m}\left(a_{\alpha}-a_{\alpha}^{j}\right) D^{\alpha} \dot{u}_{j}
$$

To obtain convergence, we will use the estimate:

$$
\begin{aligned}
\left\|\left(A-A^{j}\right) u_{j}\right\|_{L^{p}(\Omega)} \leqslant & C \sup _{|\alpha|=2 m}\left|a_{\alpha}-a_{\alpha}^{j}\right|\left\|u_{j}\right\|_{W^{2 m, p}(\Omega)} \\
& +\sum_{|\alpha|<2 m}\left\|a-a^{j}\right\|_{L^{p^{\prime}}(\Omega \cap B)}\left\|u_{j}\right\|_{W^{2 m-1, p}{ }_{(\Omega \cap B)}} \\
& +C\left\|u_{j}\right\|_{W^{2 m-1, p}(\Omega-B)}
\end{aligned}
$$

where $B$ is a bounded set. In the second term on the right, we have used the

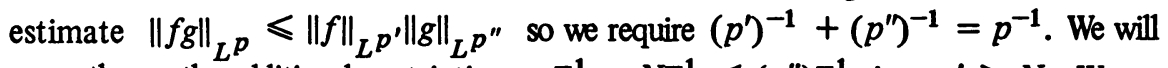
presently use the additional restriction $p^{-1}-N^{-1} \leqslant\left(p^{\prime \prime}\right)^{-1}$, i.e., $p^{\prime} \geqslant N$. We proceed to show the right side of (4.4) can be made small.

First we write the $L^{p}$ estimate (see Friedman [7, p. $68 \mathrm{ff}$.], or the remark at the end of $\S 3$ ):

$$
\left\|u_{j}\right\|_{W^{2 m, p}(\Omega)} \leqslant C\left\|\left(A^{j}+z\right) u_{j}\right\|_{L^{p}(\Omega)} \leqslant C\|f\|_{L^{p}(\Omega)}
$$

where $C$ is independent of $j$.

To make the last term of (4.4) small, we choose $B$ to be a large set. For example, let $B=B(0, R)$. Let $\psi$ be a nonnegative $C^{\infty}$ function which equals 0 in $B(0,1 / 2)$ and 1 outside $B(0,1)$. Define $\psi_{R}(x)=\psi(x / R)$. Then

$$
\left\|u_{j}\right\|_{W^{2 m-1, p}(\Omega-B)} \leqslant\left\|\psi_{R} u_{j}\right\|_{W^{2 m, p}(\Omega)} .
$$

Applying the $L^{p}$ estimate to $A^{j}+z$ and the function $\psi_{R} u_{j}$,

$$
\left\|\psi_{R} u_{j}\right\|_{W^{2 m, p}(\Omega)} \leqslant C\left\|\left(A^{j}+z\right) \psi_{R} u_{j}\right\|_{L^{p}(\Omega)}
$$

where $C$ does not depend on $j$. Since

$$
A^{j} \psi_{R} u_{j}=\psi_{R} A^{j} u_{j}+\sum_{|\alpha| \leqslant 2 m, \beta<\alpha} a_{\alpha}\left(\begin{array}{l}
\alpha \\
\beta
\end{array}\right) D^{\alpha-\beta} \psi_{R} D^{\beta} u
$$


and

$$
\left|D^{\alpha-\beta} \psi_{R}\right| \leqslant C R^{-(|\alpha-\beta|)}
$$

we get

$$
\left\|\left(A^{j}+z\right) \psi_{R} u_{j}\right\|_{L^{p}(\Omega)} \leqslant\left\|\psi_{R}\left(A^{j}+z\right) u_{j}\right\|_{L^{p}(\Omega)}+C R^{-1}\left\|u_{j}\right\|_{W^{2 m-1, p}(\Omega)} .
$$

Since $\left(A^{j}+z\right) u_{j}=f$ has compact support, we can choose $R$ large enough that the first term above is zero, and the second term is $\leqslant 1 / 2 \delta$ (recalling (4.5)).

Now with $R$ chosen, we can make the first two terms in (4.4) small. Since $a_{\alpha}^{j} \rightarrow a_{\alpha}$ uniformly for $|\alpha|=2 m$, we can again use (4.5) to show there is a $j$ large enough that the first term of (4.4) is $\leqslant 1 / 4 \delta$. Finally, since we required $p^{-1}-N^{-1} \leqslant\left(p^{\prime \prime}\right)^{-1}$,

$$
\left\|u_{j}\right\|_{W^{2 m-1, p^{\prime \prime}(\Omega \cap B)}} \leqslant C\left\|u_{j}\right\|_{W^{2 m, p}(\Omega)} \leqslant C\|f\|_{L^{p}(\Omega)} .
$$

Now $a_{\alpha}^{j} \rightarrow a_{\alpha}$ a.e. boundedly for $|\alpha|<2 m$, so $j$ can be increased to make the middle term on the right of $(4.4) \leqslant 1 / 4 \delta$. Thus we have shown

$$
\left\|\left(A-A^{j}\right) u_{j}\right\|_{L^{p}(\Omega)} \rightarrow 0 .
$$

Referring back to (4.3), we have $\left\|(A+z)\left(u_{j}-u_{k}\right)\right\|_{L^{p}(\Omega)} \rightarrow 0$. Now we can choose some value of $z$ to apply the $L^{p}$ estimate with the functions $u_{j}-u_{k}$; taking $p=q>N, u_{j}-u_{k} \rightarrow 0$ on $W^{2 m, q}(\Omega)$. Hence $u_{j}-u_{k} \rightarrow 0$ in $C^{2 m-1}(\bar{\Omega})$. So the limit function $u$ is in $C_{0}(\bar{\Omega}) \cap W^{2 m, q}(\Omega)$, satisfies null Dirichlet boundary conditions, and

$$
(A+z) u=\lim (A+z) u_{j} \text { a.e. }=\lim \left(A^{j}+z\right) u_{j} \text { a.e. }=f \text { a.e. }
$$

We may take the equality to hold everywhere, and $A u=f-z u \in C_{0}(\bar{\Omega})$. Therefore $u \in D_{0}^{q}(A)$.

Therefore $A_{0}^{q}+z$ has dense range for this particular $z$. Applying the Closed Range Theorem, then Lemma 5 again, $A_{0}^{q}+z$ maps onto for any $z$ in the sector $S_{q}$ of Theorem 1 and Lemma 5 (we momentarily emphasize the dependence of $S$ on $q$ ).

Let $q^{\prime}>q$. Then there is $z \in S_{q} \cap S_{q^{\prime}}$. It is clear from $R\left(A_{0}^{q}+z\right)=$ $R\left(A_{0}^{q^{\prime}}+z\right)=C_{0}(\bar{\Omega})$ that $D_{0}^{q}(A)=D_{0}^{q^{\prime}}(A)$.

Hence we may define an operator $A_{0}$ independent of $q$. We have from Theorem $1\left\|\left(A_{0}+z\right)^{-1}\right\|_{C(\bar{\Omega})} \leqslant M_{0}|z|^{-1}$ for $z$ in some sector $S_{q^{\prime}}$. Q.E.D.

REMARK. A similar method proves the corresponding theorem in $L^{p}$, including the case of unbounded $\Omega$ at least for $p \geqslant 2$.

We have essentially shown that $A_{0}$ generates an analytic semigroup; we 
refer to Chapter IX of Kato [9], and especially $\S 1$, par. 6 of that chapter, for the general theorems. The operator $A_{0}$ lacks only a dense domain in $C_{0}(\bar{\Omega})$; functions in this domain are zero on $\partial \Omega$, and the same is true for any uniform limit of such functions. Thus we will have to consider the space

$$
C_{00}(\bar{\Omega})=\left\{u \in C_{0}(\bar{\Omega}) \text { with } u=0 \text { on } \partial \Omega\right\}
$$

and a new domain

$$
D_{00}(A)=\left\{u \in D_{0}^{q}(A), q>N, \text { with } A u \in C_{00}(\bar{\Omega})\right\}
$$

and redefine:

$A_{00}$ is the operator with domain $D_{00}(A) \subset C_{00}(\bar{\Omega})$ and range in $C_{00}(\bar{\Omega})$ given by $A_{00} u=A u$ for $u \in D_{00}(A)$.

Also, we introduce an additional assumption on the coefficients of $A$ :

All the $a_{\alpha}$ are continuous in $\bar{\Omega}$.

THEOREM 5. Let (A1)-(A6) be satisfied, and let $\Omega$ be uniformly regular of class $C^{2 m}$, and locally regular of class $C^{2 m, \mu}, \mu>0$.

Then $-A_{00}$, as defined above, generates an analytic semigroup of bounded operators on $C_{00}(\bar{\Omega})$.

Proof. The proof of Theorem 3 is equally valid with $C_{0}(\bar{\Omega})$ replaced by $C_{00}(\bar{\Omega})$. Furthermore, we claim that the range of $A_{00}+z$ is $C_{00}(\bar{\Omega})$, so that $A_{00}$ is a closed operator, independent of $q$, with bounded inverse on $C_{00}(\bar{\Omega})$.

To show that $A_{00}+z$ maps onto $C_{00}(\bar{\Omega})$ for $z$ in the truncated sector, let $f \in C_{0}^{\infty}(\Omega)$. Then $f \in C_{0}^{\infty}(\bar{\Omega})$ and there is $u \in D_{0}^{q}(A)$ with $A u+z u=f$ according to Theorem 4. Since $A u=f-z u \in C_{00}(\bar{\Omega}), u \in D_{00}(A)$. So the range of $A_{00}+z$ is dense, and, by the closed range theorem, is all of $C_{00}(\bar{\Omega})$. $C_{00}(\bar{\Omega})$.

Finally, we observe that $A_{00}$ is defined on $C_{0}^{\infty}(\Omega)$ which is dense in

Now the estimate of Theorem 1 gives the remaining condition for the generation of an analytic semigroup. Q.E.D.

5. Applications. The fact that elliptic operators generate semigroups leads to knowledge of parabolic equations. In the case of $L^{p}$ norms, this knowledge is somewhat abstract, as the solution of $\partial u / \partial t+A u=0$ for example, can be directly analyzed only as a function $u(t)$ with values in $L^{p}(\Omega)$.

On the other hand, since the norm of $C_{00}(\bar{\Omega})$ is the sup norm, one gains in this case uniform pointwise information about $u(t, x)$ in the variable $x$. This gives valuable information about solutions in the classical sense of parabolic equations.

REMARK. Solving the equation $\partial u / \partial t+A u=f$ is equivalent to solving 
the equation

$$
\partial v / \partial t+\left(A+\lambda_{0}\right) v=e^{-\lambda_{0} t} f
$$

where $v=e^{-\lambda_{0} t} u$. Thus it is no loss of generality to replace $A$ by $A+\lambda_{0}$ in the sequel, so that $A+z$ will be invertible for any $z$ with $\operatorname{larg} z \mid \leqslant 1 / 2 \pi+\epsilon$, and in particular for $z=0$. The abstract theorems we shall quote assume this replacement has been made.

First we consider the direct application of Theorem 5 to temporally hamogeneous equations.

THEOREM 6. Consider the parabolic problem:

$$
\begin{array}{ll}
\partial u(t, x) / \partial t+A u(t, x)=f(t, x), & t>0, x \in \Omega, \\
u(0, x)=u_{0}(x), & x \in \Omega, \\
D_{x}^{\beta} u(t, x)=0, & 0 \leqslant|\beta|<m, x \in \partial \Omega,
\end{array}
$$

where $A$ is a strongly elliptic operator in $x$ satisfying the conditions (A1)-(A6), and $\Omega \subset \mathbf{R}^{N}$ is a domain uniformly of class $C^{2 m}$ and locally $C^{2 m, \mu}, \mu>0$. Suppose $u_{0} \in C_{00}(\bar{\Omega})$, and $f(t) \in C_{00}(\bar{\Omega})$ for $t \geqslant 0$; further suppose $f(t)$ is Hölder continuous in $t$ uniformly in $\bar{\Omega}$, i.e.,

$$
\sup _{x_{0} \in \bar{\Omega}}|f(t, x)-f(s, x)| \leqslant C|t-s|^{\nu}, \quad 0<\nu<1 .
$$

Then (5.1) has a solution $u(t, x)$, unique among functions with $u(t) \in$ $C_{00}(\bar{\Omega}), t \geqslant 0$, given by

$$
u(t)=U(t) u_{0}+\int_{0}^{t} U(t-s) f(s) d s
$$

where $U(t)$ is the semigroup, or abstract evolution operator coming from Theorem 5. The solution has the properties:

(i) $u(t, x)$ is continuous in $t \geqslant 0$, uniformly in $x \in \bar{\Omega}$.

(ii) $u(t, x)$ is continuously differentiable in $t>0$, uniformly in $x \in \bar{\Omega}$.

(iii) For any fixed $t>0, u(t, x) \in C^{2 m-1}(\bar{\Omega}) \cap^{\cdot} W_{\text {loc }}^{2 m, q}(\bar{\Omega}), q>N$, and $A u \in C_{00}(\bar{\Omega})$.

(iv) If $f$ is analytic in $t$, uniformly on $\bar{\Omega}$, near $[0, T], u(t, x)$ is analytic in $t$, uniformly on $\bar{\Omega}$, near $(0, T]$. If in addition the coefficients of $A$ satisfy a uniform Hölder condition in $x$, then so do all $D_{x}^{\alpha} u(t, x),|\alpha| \leqslant 2 m$, and $u(t, x)$ is a classical solution of (5.1).

Proof. From the semigroup theory (e.g., Kato [10, Theorem 1.13]), we have a solution (5.2) of the abstract evolution equation for any $f$ Hölder 
continuous in the sense

$$
\|f(t)-f(s)\| \leqslant C|t-s|^{\nu}
$$

which in $C_{00}(\bar{\Omega})$ is a hypothesis of our present theorem.

This solution $u(t)$, as a function with values in the Banach space $C_{00}(\bar{\Omega})$, is known to be strongly continuous in $t \geqslant 0$, and continuously differentiable in $t>0$. Since the norm of $C_{00}(\bar{\Omega})$ is the sup norm, this implies (i) and (ii). Similarly the first part of (iv) is just an interpretation of known abstract results. Property (iii) follows from the fact that $u(t) \in D_{00}(A)$ for any $t>0$.

To prove the rest of (iv), we can define $u(\xi, \eta, x)=u(\xi+i \eta, x)$ since $u$ extends to an analytic function in a complex neighborhood of $t$; then

$$
\Delta_{\xi \eta} u(\xi, \eta, x)=\left(\frac{\partial^{2}}{\partial \xi^{2}}+\frac{\partial^{2}}{\partial \eta^{2}}\right) u(\xi+i \eta, x)=0
$$

and $u(\xi, \eta, x)$ is a solution of the elliptic equation

$$
\left(\left(-\Delta_{\xi \eta}\right)^{m}+A+\frac{\partial}{\partial \xi}\right) u(\xi, \eta, x)=f(\xi+i \eta, x) .
$$

Therefore we can apply the interior regularity theorem for elliptic operators in a neighborhood $N_{0}$ of the point $(t, 0, x) \in \mathrm{R}^{N+2}$. The Hölder continuity of $f$ and of the coefficients of $A$ implies $u \in C^{2 m, \mu}\left(N_{0}\right), \mu>0$. In particular $u$ is a classical solution of (5.1). Q.E.D.

Now we wish to apply Theorem 5 to the case of time-dependent operators. This involves a modest investment of extra work, much of which is done in the following lemma. We introduce here the norm.

$$
N(u ; q, z)=\sup _{x_{0} \in \Omega}\|u\|_{L^{q}}\left(B\left(x_{0}, r_{z}\right) \cap \bar{\Omega}\right), \quad r_{z}=r_{0}|z|^{-1 / 2 m},
$$

and the Banach space $X_{q}=$ completion of $C_{0}^{\infty}(\Omega)$ in $N(u ; q, z)$.

Lemma 6. Let $A$ be an elliptic operator satisfying (A1)-(A6), $\Omega$ a uniformly $C^{2 m}$ domain which is locally $C^{2 m, \mu}, \mu>0$. Let $q>N$. Then $A_{0}^{q}$ can be extended to an operator $\widetilde{A}_{0}^{q}$ on the domain

$$
\begin{aligned}
& \widetilde{D}_{0}^{q}=\left\{u \in C_{00}(\bar{\Omega}), D^{\alpha} u \in X_{q},|\alpha|=2 m,\right. \\
& \text { and } \left.D^{\beta} u=0 \text { on } \partial \Omega, 0 \leqslant|\beta|<m\right\} .
\end{aligned}
$$

$\widetilde{A}_{0}^{q}$ is a closed operator from $C_{00}(\bar{\Omega})$ to $X_{q}$, and $\widetilde{A}_{0}^{q}+z$ is invertible for $\operatorname{larg} z|\leqslant 1 / 2 \pi+\epsilon| z \mid, \geqslant \lambda_{0}$. For such $z$, and $u \in \widetilde{D}_{0}^{q}$, 


$$
\begin{aligned}
|z|\|u\|_{C(\bar{\Omega})}+\sum_{0 \leqslant j<2 m}|z|^{1-j / 2 m}|[u]|_{j, C(\bar{\Omega})} & +\sum_{|\alpha|=2 m}|z|^{\theta} N\left(D^{\alpha} u ; q, z\right) \\
& \leqslant M_{0}|z|^{\theta} N((A+z) u ; q, z) .
\end{aligned}
$$

Here $M_{0}, \lambda_{0}, \epsilon$ depend on $q, \Omega, E, L, \omega$, but not on $z$.

Proof. We note that $\widetilde{D}_{0}^{q}$ does not depend on $A$, and that if $u \in \widetilde{D}_{0}^{q}$, then $A u \in \mathrm{X}_{q}$.

Recalling the proof of Theorem 2, it is clear that Theorem 2 holds for $u \in \widetilde{\mathcal{D}}_{0}^{q} ;(5.3)$ is just the estimate of Theorem 2 in new notation.

With appropriate modifications in the proofs of Theorems 3 and 4, we can show that $\widetilde{A}_{0}^{q}$ is a closed operator, with bounded resolvent for $z$ in a truncated sector. Q.E.D.

THEOREM 7. Consider the temporally inhomogeneous equation

$$
\begin{array}{ll}
\partial u(t, x) / \partial t+A(t, x) u(t, x)=f(t, x), & t>0, x \in \Omega, \\
u(0, x)=u_{0}(x), & x \in \Omega, \\
D_{x}^{\beta} u(t, x)=0, & 0 \leqslant|\beta|<m, x \in \partial \Omega,
\end{array}
$$

where for each $t \geqslant 0, A(t)$ is an elliptic operator in $x$, satisfying conditions (A1)-(A6) uniformly in $t$. The domain $\Omega$ is uniformly of class $C^{2 m}$ and locally $C^{2 m, \mu}, \mu>0$.

Suppose further that the coefficients $a_{\alpha}(t, x)$ of $A(t, x)$ have first derivatives $(\partial / \partial t) a_{\alpha}(t, x)$ which are bounded and satisfy a Hölder condition in $t$, uniformly on $t$ and $x \in \bar{\Omega}$.

Then for functions $u_{0}, f$ as in Theorem 6, the problem (5.4) has a solution $u(t, x)$, unique among functions with $u(t) \in C_{00}(\bar{\Omega}), t \geqslant 0$, having the properties (i), (ii), and (iii)-with $A(t) u(t)$ replacing $A u(t)$-of Theorem 6. Property (iv) is also valid if in addition the coefficients $a_{\alpha}(t, x)$ are assumed to be analytic in $t$, uniformly on $\bar{\Omega}$.

Proof. Under the hypotheses of the theorem, the operators $-A_{00}(t)$ generate semigroups in $C_{00}(\bar{\Omega})$ uniformly in $t$. We wish to use the abstract theory of evolution equations for operators $A(t)$ with domains depending on $t$. We again refer to Kato [10], and in particular Theorem 3.1, for which it suffices that we verify the following abstract conditions:

(1) $(\partial / \partial t)(A(t)+z)^{-1}$ exists, for $z$ in a sector $|\arg z| \leqslant 1 / 2 \pi+\epsilon$, as a bounded operator with

$$
\left\|(\partial / \partial t)(A(t)+z)^{-1}\right\| \leqslant M_{0} /|z| .
$$

(2) $(\partial / \partial t) A(t)^{-1}$ is $t$-Hölder continuous in norm. (We draw attention to 
the remark preceding Theorem 6.) To verify the conditions, we will use Lemma 6, which shows that $A_{00}(t)$ is almost equal to an operator on a domain independent of $t$.

To verify (1), we note that formally

$$
-(\partial / \partial t)(A(t)+z)^{-1} \supset(A(t)+z)^{-1} A_{t}(t)(A(t)+z)^{-1}
$$

where

$$
A_{t}(t)=\sum_{|\alpha| \leqslant 2 m}\left((\partial / \partial t) a_{\alpha}(t, x)\right) D_{x}^{\alpha} .
$$

By considering the three operators in sequence, one can show that the right side of (5.5) can be completed to an operator on all of $C_{00}(\bar{\Omega})$ satisfying the desired bound.

To do this one uses the sequence of norms (reversing the order of (5.5)):

$$
\begin{aligned}
\|\cdot\|_{C(\Omega)} & \rightarrow|z|^{\theta} \sum_{|\alpha|<2 m} N\left(D^{\alpha} \cdot ; q, z\right) \\
& \rightarrow|z|^{\theta} N(\cdot ; q, z) \rightarrow|z|^{1-\theta}|z|^{\theta}\|\cdot\|_{C(\Omega)} .
\end{aligned}
$$

In the last step, Lemma 6 is applied.

To verify (2), we again refer to (5.5) with $z=0$. We then show that each of the three (extended) operators on the right is Hölder continuous in $t$, independent of $f$, using the norms of (5.6).

In fact, $A_{t}(t)$ is clearly Hölder continuous in view of the assumptions on the $a_{\alpha}(t, x)$.

For the first and last operators in the sequence, we choose $f \in X_{q}$, and let $v(t), v(s)$ be solutions of

$$
A(t) v(t)=f, \quad A(s) v(s)=f
$$

with zero Dirichlet boundary data. Estimates for $|v(s)-v(t)|$ can then be derived, making use of the fact that the domain in Lemma 6 is independent of the operator; hence, for example, both $v(t)$ and $v(s)$ are in the domain of $A(t)$ as defined there.

Thus one verifies the hypotheses in the theorem on abstract evolution equations. Hence there is a unique abstract solution of the equation in the present case. The concrete properties of $u(t, x)$ follow using arguments like those of Theorem 6. Q.E.D.

\section{BIBLIOGRAPHY}

1. S. Agmon, On the eigenfunctions and on the eigenvalues of general elliptic boundary value problems, Comm. Pure Appl. Math. 15 (1962), 119-147. MR 26 \#5288.

2. - Lectures on elliptic boundary value problems, Van Nostrand Math. Studies, no. 2, Van Nostrand, Princeton, N. J., 1965. MR 31 \#2504. 
3. S. Agmon, A. Douglis and L. Nirenberg, Estimates near the boundary for solutions of elliptic partial differential equations satisfying general boundary conditions. I, Comm. Pure , Appl. Math. 12 (1959), 623-727. MR 23 \#A2610.

4. R. Arima, On general boundary value problems for parabolic equations, J. Math. Kyoto Univ. 4 (1964), 207-243. MR 33 \#6156.

5. F. E. Browder, On the spectral theory of elliptic differential operators. I, Math. Ann. 142 (1960/61), 22-130. MR 35 \#804.

6. S. D. Ė̌̀del'man, Parabolic systems, "Nauka", Moscow, 1964; English transl., Noordhoff, Groningen; North-Holland, Amsterdam, 1969. MR 29 \#4998; 40 \#6023.

7. A. Friedman, Partial differential equations, Holt, Rinehart and Winston, New York, 1969.

8. E. Hille and R. S. Phillips, Functional analysis and semi-groups, rev. ed., Amer. Math. Soc. Colloq. Publ., vol. 31, Amer. Math. Soc., Providence, R. I., 1957. MR 19, 664.

9. T. Kato, Perturbation theory for linear operators, Die Grundlehren der math. Wissenschaften, Band 132, Springer-Verlag, New York, 1966. MR 34 \#3324.

10. Semigroups and temporally inhomogeneous evolution equations, C.I.M.E. lecture notes, Varenna, 1963.

11. T. Kato and H. Tanabe, On the abstract evolution equation, Osaka Math. J. 14 (1962), 107-133. MR 25 \#4367.

12. - On the analyticity of solution of evolution equations, Osaka J. Math. 4 (1967), 1-4. MR 36 \#5482.

13. J. L. Lions and E. Magenes, Problèmes aux limites non homogènes et applications. Vol. 1, Travaux et Recherches Mathématiques, no. 17, Dunod, Paris, 1968. MR 40 \#512.

14a. K. Masuda, Manuscript for seminar at Kyoto Univ., 1970.

14b. - Manuscript, 1972.

15. C. B. Morrey, Jr., Multiple integrals in the calculus of variations, Die Grundlehren der math. Wissenschaften, Band 130, Springer-Verlag, New York, 1966. MR 34 \#2380.

16. H. Tanabe, On Green's functions of elliptic and parabolic boundary value problems, Proc. Japan Acad. 48 (1972), 709-711.

17. K. Yosida, Functional analysis, 2 nd ed., Die Grundlehren der math. Wissenschaften, Band 123, Academic Press, New York; Springer-Verlag, Berlin, 1968. MR 39 \#741.

DEPARTMENT OF MATHEMATICS, UNIVERSITY OF CALIFORNIA, BERKELEY, CALIFORNIA 94720

INSTITUTE FOR NEUTRON PHYSICS AND REACTOR TECHNOLOGY, KARLSRUHE NUCLEAR RESEARCH CENTER, KARLSRUHE, FEDERAL REPUBLIC OF GERMANY 